\title{
UNIVERSALLY FIRST RETURN CONTINUOUS FUNCTIONS
}

\author{
UDAYAN B. DARJI, MICHAEL J. EVANS, AND RICHARD J. O'MALLEY
}

(Communicated by Andrew Bruckner)

\begin{abstract}
It is known that the first return continuous functions are precisely the Darboux functions in Baire class 1, and that every such function can be changed via a homeomorphism into an approximately continuous function. Here we give two characterizations of the smaller class of universally first return continuous functions, one of which is the capacity of changing such a function via a homeomorphism into an approximately continuous function which is continuous almost everywhere.
\end{abstract}

O'Malley [4] introduced the notion of first return path systems to study differentiation properties of real-valued functions defined on the unit interval. In [1] the present authors showed that a large class of functions, including the approximately differentiable functions, are universally first return differentiable. In that same paper the notions of first return continuity and universal first return continuity were introduced, and first return continuous functions were shown to be the familiar Darboux, Baire 1 functions. Here we give two characterizations of the proper subclass of the Darboux, Baire 1 functions consisting of the universally first return continuous functions. We shall show that $f:[0,1] \rightarrow \mathbb{R}$ is universally first return continuous if and only if $f$ is Darboux, Baire 1 and the graph of $f$ restricted to $C(f)$, the set of points of continuity of $f$, is dense in the entire graph. We let $\mathscr{G}$ denote this collection of all Darboux, Baire 1 functions $f:[0,1] \rightarrow \mathbb{R}$ for which $f \mid C(f)$ is dense in $f$. Recalling that the Maximoff-Preiss Theorem (see [5], Theorem 2) states that a function is Darboux, Baire 1 if and only if there is a homeomorphism $h$ of $[0,1]$ onto itself such that $f \circ h$ is approximately continuous, it seems natural to inquire if we can obtain an even nicer function via a homeomorphism starting with a function in $\mathscr{G}$, and perhaps characterizing the class $\mathscr{G}$ in this manner. We show that this is, indeed, possible.

Before proving our results, we need to review some terminology from [1] and [3]. By a trajectory we mean any sequence $\left\{x_{n}\right\}_{n=0}^{\infty}$ of distinct points in $(0,1)$, which is dense in $[0,1]$. One method of specifying a trajectory is to assign an enumeration or ordering to a given countable dense subset $D$ of $(0,1)$.

Received by the editors April 23, 1993 and, in revised form, August 31, 1993.

1991 Mathematics Subject Classification. Primary 26A21; Secondary 26A24.

Key words and phrases. First return continuity, approximate continuity, a.e. topology, Baire class one, Darboux. 
Throughout we shall refer to such a set $D$ as a support set. Let $\left\{x_{n}\right\}$ be a fixed trajectory. For a given interval $(a, b) \subset[0,1], r(a, b)$ will be the first element of the trajectory in $(a, b)$. For $0 \leq y<1$, the right first return path to $y$, $R_{y}^{+}$, is defined recursively via

$$
y_{1}^{+}=1 \text { and } y_{k+1}^{+}=r\left(y, y_{k}^{+}\right) .
$$

For $0<y \leq 1$, the left first return path to $y, R_{y}^{-}$, is defined similarly. For $0<y<1$, we set $R_{y}=R_{y}^{+} \cup R_{y}^{-} \cup\{y\}, R_{0}=\{0\} \cup R_{0}^{+}$, and $R_{1}=R_{1}^{-} \cup\{1\}$. The collection $\mathscr{R} \equiv\left\{R_{y}: y \in[0,1]\right\}$ forms a path system, which we shall refer to as the first return path system determined by the trajectory $\left\{x_{n}\right\}$. Let $f:[0,1] \rightarrow \mathbb{R}$. We say that $f$ is first return continuous on $[0,1]$ with respect to a trajectory $\left\{x_{n}\right\}$ provided that at each $y \in[0,1]$ we have

$$
\lim _{\substack{t \rightarrow y \\ t \in R_{y} \backslash\{y\}}} f(t)=f(y) .
$$

The function $f$ is said to be first return continuous on $[0,1]$ if there exists a trajectory $\left\{x_{n}\right\}$ such that $f$ is first return continuous on $[0,1]$ with respect to $\left\{x_{n}\right\}$. Finally, $f$ is said to be universally first return continuous on $[0,1]$, if for every support set $D$ there is an ordering $\left\{x_{n}\right\}$ of $D$, such that $f$ is first return continuous on $[0,1]$ with respect to $\left\{x_{n}\right\}$.

A set $U$ is said to be almost open if it has density one at all of its points and if $\lambda(U)=\lambda\left(U^{o}\right)$, where $l$ denotes Lebesgue measure and $U^{o}$ denotes the interior of $U$. The collection of all almost open sets forms what is called the a.e. topology. The collection of all functions which are continuous in this topology is precisely the collection of all approximately continuous functions which are continuous almost everywhere [3, Theorem 3.2].

Before proving our characterizations of universally first return continuous functions, we present a result, Theorem 1, concerning first return continuous functions which may be of independent interest. We first state a simple lemma, which was also stated in [1], in which we use the symbol $d(y,[a, b])$ to denote the distance from a point $y$ to an interval $[a, b]$. (The straightforward proof is omitted.) Then Lemma 2 contains the heart of the proof of Theorem 1.

Lemma 1. Suppose $F$ is differentiable at $y$ and $\epsilon>0$ and $y$ is contained in some closed interval $I$ such that if $t \in I \backslash\{y\}$, then $\left|\frac{F(t)-F(y)}{t-y}-F^{\prime}(y)\right| \leq \epsilon$. If $[a, b] \subset I$, then

$$
\left|\frac{F(a)-F(b)}{a-b}-F^{\prime}(y)\right| \leq \epsilon \cdot\left(1+2 \cdot \frac{d(y,[a, b])}{|a-b|}\right) .
$$

Lemma 2. Let $f:[0,1] \rightarrow \mathbb{R}$ be a derivative and $D$ a support set such that $f \mid D$ is dense in $f$. Then there is an ordering $\left\{x_{n}\right\}$ of $D$ such that $f$ is first return continuous with respect to the trajectory $\left\{x_{n}\right\}$.

Proof. Let $D$ be the given support set for which $f \mid D$ is dense in $f$, and let $\left\{d_{m}\right\}_{m=0}^{\infty}$ be an ordering of $D$. We will inductively construct a sequence of partitions $\left\{\mathscr{P}_{k}\right\}$,

$$
\mathscr{P}_{k}=\left\{p_{0}^{k}=0<p_{1}^{k}<\cdots<p_{l_{k}}^{k}=1\right\}
$$


where each $\mathscr{P}_{k}$ consists of points chosen from $D \cup\{0,1\}$, and each $\mathscr{P}_{k+1}$ is a refinement of $\mathscr{P}_{k}$. At the same time, we will define an ordering $\left\{x_{n}\right\}_{n=0}^{\infty}$ of $D$ utilizing $\left\{\mathscr{P}_{k}\right\}$. We shall start by setting $\mathscr{P}_{0}=\{0,1\}$ and setting $x_{-2}=0$ and $x_{-1}=1$. In general we label the points in $\mathscr{P}_{k+1} \backslash \mathscr{P}_{k}$ from left to right as $x_{l_{k}-1}, x_{l_{l}}, \ldots, x_{l_{k+1}-2}$. We shall show that $f$ is first return continuous with respect to the trajectory $\left\{x_{n}\right\}$.

As $\left\{\mathscr{P}_{k}\right\}$ is defined, we shall also define a strictly increasing sequence $\left\{n_{m}\right\}_{m=-1}^{\infty}$ of integers, starting with $n_{-1}=-1$, and for $m \geq 0, n_{m}$ will be the smallest value of $n>n_{m-1}$ such that $d_{m} \in \mathscr{P}_{n}$. (Of course, part of our proof will be to show that such an $n_{m}$ exists.) As each $n_{m}$ is defined, the process for constructing the succeeding $\mathscr{P}_{n}$ 's, for $n>n_{m}$, is altered slightly.

For each $y \in(0,1]$ and each non-negative integer $k$ we let $\lambda^{k}(y)$ denote the closest element of the partition $\mathscr{P}_{k}$ lying strictly to the left of $y$; and for each $y \in[0,1)$ and each non-negative integer $k$ we shall let $\rho^{k}(y)$ denote the closest element of the partition $\mathscr{P}_{k}$ lying strictly to the right of $y$. For convenience, we let $\lambda^{-1}(y)=-1$ and $\rho^{-1}(y)=2$ for all $y \in[0,1]$. Let $F$ be such that $F^{\prime}=f$. For each $y \in[0,1]$ and each closed interval $I$ containing $y$ in its interior, we set

$$
\Delta(F, y, I)=\sup \left\{\left|\frac{F(x)-F(y)}{x-y}-F^{\prime}(y)\right|: x \in I \backslash\{y\}\right\} .
$$

For each non-negative integer $m$, and each integer $n>n_{m-1}$, we want $\mathscr{P}_{n}$ to satisfy the following conditions:

(1) If $y \in(0,1], t \in\left(\lambda^{n-1}(y), \lambda^{n}(y)\right] \cap \mathscr{P}_{n}$, and $t>0$, then $|f(t)-f(y)| \leq$ $5 \cdot \Delta\left(F, y,\left[\lambda^{n-1}(y), \rho^{n-1}(y)\right]\right)+1 /(m+1)$.

(2) If $y \in[0,1), t \in\left[\rho^{n}(y), \rho^{n-1}(y)\right) \cap \mathscr{P}_{n}$, and $t<1$, then $|f(t)-f(y)| \leq$ $5 \cdot \Delta\left(F, y,\left[\lambda^{n-1}(y), \rho^{n-1}(y)\right]\right)+1 /(m+1)$.

(3) And, $\operatorname{mesh}\left(\mathscr{P}_{n}\right) \leq\left(\frac{2}{3}\right)^{n}$.

As noted previously, we start the process by setting $\mathscr{P}_{0}=\left\{p_{0}^{0}=0, p_{1}^{0}=1\right\}$, and $x_{-2}=0$ and $x_{-1}=1$. Note that $\mathscr{P}_{0}$ satisfies conditions (1), (2), and (3), with $m=0$. (It satisfies (1) and (2) vacuously.) Suppose that $\mathscr{P}_{k}$ has been defined and satisfies conditions (1), (2), and (3), and yet $d_{0} \notin \mathscr{P}_{k}$. Say $\mathscr{P}_{k}=\left\{p_{0}^{k}=0<p_{1}^{k}<\cdots<p_{l_{k}}^{k}=1\right\}$. Since $\mathscr{P}_{k+1}$ has to be a refinement of $\mathscr{P}_{k}$, put all points of $\mathscr{P}_{k}$ in $\mathscr{P}_{k+1}$. For each $0 \leq i \leq l_{k}-1$, we will select points which properly refine $\left\{p_{i}^{k}, p_{i+1}^{k}\right\}$. Fix such an $i$ and let $l_{i}^{k}=\frac{2}{3} p_{i}^{k}+\frac{1}{3} p_{i+1}^{k}$ and $r_{i}^{k}=\frac{1}{3} p_{i}^{k}+\frac{2}{3} p_{i+1}^{k}$. Using the mean value theorem, obtain $p_{i, l}^{k} \in\left(p_{i}^{k}, l_{i}^{k}\right)$, $p_{i, m}^{k} \in\left(l_{i}^{k}, r_{i}^{k}\right)$, and $p_{i, r}^{k} \in\left(r_{i}^{k}, p_{i+1}^{k}\right)$ such that

$$
\begin{gathered}
f\left(p_{i, l}^{k}\right)=F^{\prime}\left(p_{i, l}^{k}\right)=\frac{F\left(p_{i}^{k}\right)-F\left(l_{i}^{k}\right)}{p_{i}^{k}-l_{i}^{k}}, \\
f\left(p_{i, m}^{k}\right)=F^{\prime}\left(p_{i, m}^{k}\right)=\frac{F\left(l_{i}^{k}\right)-F\left(r_{i}^{k}\right)}{l_{i}^{k}-r_{i}^{k}},
\end{gathered}
$$

and

$$
f\left(p_{i, r}^{k}\right)=F^{\prime}\left(p_{i, r}^{k}\right)=\frac{F\left(r_{i}^{k}\right)-F\left(p_{i+1}^{k}\right)}{r_{i}^{k}-p_{i+1}^{k}}
$$


Next, let $d_{i, l}^{k}$ denote the point $d_{s} \in\left(p_{i}^{k}, l_{i}^{k}\right]$ with minimal subscript $s$ for which $\left|f\left(p_{i, l}^{k}\right)-f\left(d_{s}\right)\right|<1$. Similarly, let $d_{i, m}^{k}$ denote the point $d_{s} \in\left(l_{i}^{k}, r_{i}^{k}\right]$ with minimal subscript $s$ for which $\left|f\left(p_{i, m}^{k}\right)-f\left(d_{s}\right)\right|<1$; and let $d_{i, r}^{k}$ denote the point $d_{s} \in\left(p_{i}^{k}, p_{i+1}^{k}\right]$ with minimal subscript $s$ for which $\mid f\left(p_{i, r}^{k}\right)-$ $f\left(d_{s}\right) \mid<1$.

We put all points of the form $d_{i, l}^{k}, d_{i, m}^{k}$, and $d_{i, r}^{k}, 0 \leq i \leq l_{k-1}$, in the partition $\mathscr{P}_{k+1}$ and order it in the natural increasing fashion as $\mathscr{P}_{k}=\left\{p_{0}^{k+1}=\right.$ $\left.0<p_{1}^{k+1}<\cdots<p_{l_{k+1}}^{k+1}=1\right\}$. We also label points in $\mathscr{P}_{k+1} \backslash \mathscr{P}_{k}$ from left to right as $x_{l_{k}-1}, x_{l_{k}}, \ldots, x_{l_{k+1}-2}$.

We need to show that conditions (1)-(3) are satisfied by $\mathscr{P}_{k+1}$, still with $m=$ 0 . Let us first consider condition (1). Let $y \in(0,1], t \in\left(\lambda^{k}(y), \lambda^{k+1}(y)\right] \cap$ $\mathscr{P}_{k+1}$, and $t>0$. Let $i$ be such that $p_{i}^{k}<y \leq p_{i+1}^{k}$. Then $t$ has to be one of $d_{i, l}^{k}, d_{i, m}^{k}$, or $d_{i, r}^{k}$. Let us first assume that $t=d_{i, l}^{k}$. Now we have two cases to consider: $y \in\left[p_{i}^{k}, l_{i}^{k}\right]$ or $y \notin\left[p_{i}^{k}, l_{i}^{k}\right]$. If $y \in\left[p_{i}^{k}, l_{i}^{k}\right]$, then

$$
\begin{aligned}
|f(t)-f(y)| & =\left|f\left(d_{i, l}^{k}\right)-f(y)\right| \leq\left|f\left(d_{i, l}^{k}\right)-f\left(p_{i, l}^{k}\right)\right|+\left|f\left(p_{i, l}^{k}\right)-f(y)\right| \\
& <1+\left|\frac{F\left(p_{i}^{k}\right)-F\left(l_{i}^{k}\right)}{p_{i}^{k}-l_{i}^{k}}-f(y)\right| \leq 1+\Delta\left(F, y,\left[\lambda^{k}(y), \rho^{k}(y)\right]\right) .
\end{aligned}
$$

If $y \notin\left[p_{i}^{k}, l_{i}^{k}\right]$, then by Lemma 1 , we have that

$$
\begin{aligned}
|f(t)-f(y)| & =\left|f\left(d_{i, l}^{k}\right)-f(y)\right| \leq\left|f\left(d_{i, l}^{k}\right)-f\left(p_{i, l}^{k}\right)\right|+\left|f\left(p_{i, l}^{k}\right)-f(y)\right| \\
& <1+\left|\frac{F\left(p_{i}^{k}\right)-F\left(l_{i}^{k}\right)}{p_{i}^{k}-l_{i}^{k}}-f(y)\right| \\
& \leq 1+\Delta\left(F, y,\left[\lambda^{k}(y), \rho^{k}(y)\right]\right) \cdot\left(1+2 \cdot \frac{d\left(y,\left[p_{i}^{k}, l_{i}^{k}\right]\right)}{\left|p_{i}^{k}-l_{i}^{k}\right|}\right) .
\end{aligned}
$$

Since $d\left(y,\left[p_{i}^{k}, l_{i}^{k}\right]\right) \leq \frac{2}{3}\left|p_{i}^{k}-p_{i+1}^{k}\right|$ and $\left|p_{i}^{k}-l_{i}^{k}\right|=\frac{1}{3}\left|p_{i}^{k}-p_{i+1}^{k}\right|$, we have that

$$
|f(t)-f(y)| \leq 1+5 \cdot \Delta\left(F, y,\left[\lambda^{k}(y), \rho^{k}(y)\right]\right) \text {. }
$$

If $t=d_{i, m}^{k}$ or $t=d_{i, r}^{k}$, we may also obtain by an argument similar to the above that $|f(t)-f(y)| \leq 1+5 \cdot \Delta\left(F, y,\left[\lambda^{k}(y), \rho^{k}(y)\right]\right)$. We just consider the interval $\left[l_{i}^{k}, r_{i}^{k}\right]$ if $t=d_{i, m}^{k}$, and the interval $\left[r_{i}^{k}, p_{i+1}^{k}\right]$ if $t=d_{i, r}^{k}$. Thus, condition (1) holds. Condition (2) may be verified by a symmetric argument.

That $\operatorname{mesh}\left(\mathscr{P}_{k+1}\right) \leq\left(\frac{2}{3}\right)^{k+1}$ easily follows from the induction hypothesis and the facts that for every $0 \leq i \leq l_{k-1}$, each of $\left|p_{i}^{k}-d_{i, l}^{k}\right|$ and $\left|p_{i+1}^{k}-d_{i, r}^{k}\right|$ is less than $\frac{1}{3} \cdot\left|p_{i}^{k}-p_{i+1}^{k}\right|$, and each of $\left|d_{i, l}^{k}-d_{i, m}^{k}\right|$ and $\left|d_{i, m}^{k}-d_{i, r}^{k}\right|$ is less than $\frac{2}{3} \cdot\left|p_{i}^{k}-p_{i+1}^{k}\right|$. Thus, condition (3) is satisfied.

If $d_{0} \notin \mathscr{P}_{k+1}$, then we continue in this manner to obtain $\mathscr{P}_{k+2}$. In so doing, we must encounter a smallest $n$ such that $d_{0} \in \mathscr{P}_{n}$. This is simply because the mesh of our partitions is decreasing to zero, and $F$ is differentiable at $d_{0}$ with derivative $f\left(d_{0}\right)$; so that at some stage $d_{0}$ must be chosen as one of $d_{i, l}^{n-1}$, $d_{i, m}^{n-1}$, or $d_{i, r}^{n-1}$. We then set $n_{0}$ equal to this $n$. 
Now assume that $j$ is a non-negative integer, that $n_{j}$ has been defined, that partition $\mathscr{P}_{n_{j}}$ has been defined satisfying conditions (1), (2), and (3) (with $m=j$ ), and $d_{j} \in \mathscr{P}_{n_{j}}$. We define $\mathscr{P}_{n_{j}+1}$ as a refinement of $\mathscr{P}_{n_{j}}$ in exactly the same manner that we described above for obtaining $\mathscr{P}_{k+1}$ as a refinement of $\mathscr{P}_{k}$ except that where we used the estimate " 1 " there, we now use the estimate “ $\frac{1}{j+2}$ ". That $\mathscr{P}_{n_{j}+1}$ satisfies conditions (1), (2), and (3) with $m=j+1$ follows precisely as before. We continue refining the partitions in this manner until we encounter the smallest integer $n>n_{j}$ for which $d_{j+1} \in \mathscr{P}_{n}$. (That we must encounter such an integer $n$ follows from the same reasoning that showed the existence of $n_{0}$. ) We then set $n_{j+1}$ equal to this value of $n$. (If $d_{j+1} \in \mathscr{P}_{k}$ for some $k \leq n_{j}$, then $n_{j+1}=n_{j}+1$.)

This completes our construction of the sequence $\left\{\mathscr{P}_{k}\right\}$ and the associated trajectory $\left\{x_{n}\right\}$. (Clearly, the range of $\left\{x_{n}\right\}$ is precisely $D \cup\{0,1\}$, so that this is indeed a trajectory.)

Now we want to show that $f$ is first return continuous with respect to this trajectory at each point. Let $y \in(0,1]$. First, observe that the left first return path to $y$ is

$$
R_{y}^{-}=\bigcup_{k=0}^{\infty}\left\{\left(\lambda^{k-1}(y), \lambda^{k}(y)\right] \cap \mathscr{P}_{k}\right\} .
$$

Let $\epsilon>0$. Let $\delta>0$ such that $y-\delta>0$, and if $0<|t-y|<\delta$, then $\left|\frac{F(t)-F(y)}{t-y}-f(y)\right|<\frac{\epsilon}{12}$. Choose $m$ so large that $\frac{1}{m+1}<\frac{\epsilon}{2}$ and let $n$ be a positive integer greater than $n_{m-1}$ such that $\left(\frac{2}{3}\right)^{n}<\frac{\delta}{2}$. Then, by condition (1) of the induction hypothesis, we have that if $k>n$, and $t \in\left\{\left(\lambda^{k-1}(y), \lambda^{k}(y)\right] \cap \mathscr{P}_{k}\right\}$, then

$$
|f(t)-f(y)| \leq 5 \cdot \Delta\left(F, y,\left[\lambda^{k-1}(y), \rho^{k-1}(y)\right]\right)+1 /(m+1)<5 \frac{\epsilon}{12}+\frac{\epsilon}{2}<\epsilon .
$$

Consequently, $|f(t)-f(y)|<\epsilon$ for all $t \in \bigcup_{k=n+1}^{\infty}\left\{\left(\lambda^{k-1}(y), \lambda^{k}(y)\right] \cap \mathscr{P}_{k}\right\}$. Thus, we have that $f$ is left first return continuous with respect to $\left\{x_{n}\right\}$. A symmetric argument also shows that $f$ is right first return continuous with respect to $\left\{x_{n}\right\}$ at each point of $[0,1)$.

Theorem 1. Let $f:[0,1] \rightarrow \mathbb{R}$ be a Darboux, Baire class 1 function and let $D$ be any support set for which $f \mid D$ is dense in $f$. Then there is an ordering $\left\{x_{n}\right\}$ of $D$ such that $f$ is first return continuous with respect to the trajectory $\left\{x_{n}\right\}$.

Proof. By the Maximoff-Preiss Theorem ([2], [5]), there exists a derivative $g$ : $[0,1] \rightarrow \mathbb{R}$ and a homeomorphism $h$ of $[0,1]$ onto itself such that $f=g \circ h$. Clearly $h(D)$ is a support set and $g \mid h(D)$ is dense in $g$. Hence, by Lemma 2, there is an ordering $\left\{h\left(x_{n}\right)\right\}$ of $h(D)$ such that $g$ is first return continuous with respect to the trajectory $\left\{h\left(x_{n}\right)\right\}$. It readily follows that $f$ is first return continuous with respect to the trajectory $\left\{x_{n}\right\}$.

Theorem 2. For a function $f:[0,1] \rightarrow \mathbb{R}$ the following are equivalent:

(1) $f \in \mathscr{G}$.

(2) $f$ is universally first return continuous.

(3) There exists a trajectory $\left\{x_{n}\right\} \subseteq(0,1) \cap C(f)$ such that $f$ is first return continuous with respect to $\left\{x_{n}\right\}$. 
(4) There exists an increasing homeomorphism $h$ of $[0,1]$ onto itself such that $f \circ h$ is continuous in the a.e. topology.

Proof. We first show that $(1) \Rightarrow(2)$ : Suppose that $f$ is a Darboux function of Baire class 1 with the property that $f \mid C(f)$ is dense in $f$. Let $D$ be an arbitrary support set. Since $f \mid C(f)$ is dense in $f$, it is an easy matter to see that $f \mid D$ is dense in $f$ as well, and we may apply Theorem 1 to complete the proof of this part.

That $(2) \Rightarrow(3)$ is obvious.

We next show that $(3) \Rightarrow(4)$ : We shall construct this homeomorphism by constructing a countable dense subset $Q$ of $[0,1]$ and defining $g$ to be an increasing function on $Q$ such that $g(Q)$ is dense in $[0,1]$. Extending $g$ to all of $[0,1]$ and letting $h=g^{-1}$, we will show that $h$ has the desired properties.

To this end, let $D$ denote the range of the given trajectory $\left\{x_{n}\right\} \subseteq(0,1) \cap$ $C(f)$, with respect to which $f$ is first return continuous. For each $n$ we let $\mathscr{P}_{n}$ denote the partition $\left\{x_{-2}=0, x_{-1}=1, x_{0}, x_{1}, \ldots, x_{n}\right\}$ of $[0,1]$. We shall enlarge each partition $\mathscr{P}_{n}$ to a partition $\mathscr{Q}_{n}=\left\{x_{-2}=0, x_{-1}=\right.$ $\left.1, a_{0}, b_{0}, x_{0}, a_{1}, b_{1}, x_{1}, \ldots, a_{n}, b_{n} x_{n}\right\}$ and define $g$ on $\mathscr{Q}_{n}$ so that for each $n$

(a) $x_{n} \in\left(a_{n}, b_{n}\right)=I_{n}$ with $a_{n}, b_{n} \in C(f) \backslash D$.

(b) If $j<n$ and $I_{j} \cap I_{n} \neq \emptyset$, then $\overline{I_{n}} \subset I_{j}$.

(c) osc $f \mid I_{n}<1 /(n+1)$.

(d) $I_{n}$ is a proper subset of the same partitioning interval of $\mathscr{P}_{n-1}$ as $x_{n}$.

(e) $g$ is increasing on $\mathscr{Q}_{n}$.

(f) If $q_{n-1}$ and $\hat{q}_{n-1}$ are the nearest elements of $\mathscr{Q}_{n-1}$ left and right, respectively, of $x_{n}$, then

$$
\frac{g\left(b_{n}\right)-g\left(a_{n}\right)}{g\left(\hat{q}_{n-1}\right)-g\left(q_{n-1}\right)} \geq 1-\frac{1}{2^{n+1}} .
$$

Starting with $n=0$, consider the partition $\mathscr{P}_{0}=\left\{0=x_{-2}<x_{0}<x_{-1}=1\right\}$. Select $I_{0}=\left(a_{0}, b_{0}\right)$ such that $x_{0} \in I_{0}$, osc $f \mid I_{0}<1$, and both $a_{0}$ and $b_{0}$ belong to $C(f) \backslash D$. Then set $\mathscr{Q}_{1}=\left\{0=x_{-2}<a_{0}<x_{0}<b_{0}<x_{-1}=1\right\}$ and set $g(0)=0, g(1)=1, g\left(x_{0}\right)=1 / 2, g\left(a_{0}\right)=1 / 4$, and $g\left(b_{0}\right)=3 / 4$. We have now defined $g$ as an increasing function on $\mathscr{Q}_{0}$, and have clearly satisfied condition (a) through (f) with $n=0$. (In (d) and (f), consider $\mathscr{P}_{-1}=\mathscr{Q}_{-1}=$ $\{0,1\}$.

For any $n \geq 0$, assume that $\mathscr{Q}_{n}=\left\{x_{-2}, x_{-1}, a_{0}, b_{0}, x_{0}, a_{1}, b_{1}, x_{1}, \ldots\right.$, $\left.a_{n}, b_{n}, x_{n}\right\}$ has been defined and $g$ has been defined on $\mathscr{Q}_{n}$ with (a) through (f) satisfied. Select $I_{n+1}=\left(a_{n+1}, b_{n+1}\right) \subseteq \overline{\left(a_{n+1}, b_{n+1}\right)} \subseteq\left(q_{n}, \hat{q}_{n}\right)$ so that $x_{n+1} \in$ $I_{n+1}$, osc $f \mid I_{n+1}<1 /(n+2)$, and both $a_{n+1}$ and $b_{n+1}$ belong to $C(f) \backslash D$. Define

$$
\begin{gathered}
g\left(x_{n+1}\right)=\frac{g\left(q_{n}\right)+g\left(\hat{q}_{n}\right)}{2}, \\
g\left(a_{n+1}\right)=g\left(q_{n}\right)+\frac{g\left(\hat{q}_{n}\right)-g\left(q_{n}\right)}{2^{n+3}},
\end{gathered}
$$

and

$$
g\left(b_{n+1}\right)=g\left(\hat{q}_{n}\right)-\frac{g\left(\hat{q}_{n}\right)-g\left(q_{n}\right)}{2^{n+3}} .
$$


It clearly follows that conditions (a) through (f) are satisfied.

Now let $Q=\bigcup_{n=0}^{\infty} Q_{n}$. Thus, $Q$ is dense in $[0,1], g$ is increasing on $Q$, and we may extend $g$ to an increasing function on all of $[0,1]$. We need to show that $g(Q)$ is dense in $[0,1]$. If $[c, d]$ is a partitioning interval of $Q_{n}$, there will be a first $x_{m}, m>n$, for which $x_{m} \in(c, d)$. Then $g\left(x_{m}\right)$ is the midpoint of the partitioning interval $[g(c), g(d)]$ of $g\left(Q_{n}\right)$. Thus, we are assured that in finitely many stages, we will encounter a $j>n$ such that the mesh of the partition $g\left(Q_{j}\right)$ will be no more than half the mesh of $g\left(Q_{n}\right)$, and therefore, $g(Q)$ is dense in $[0,1]$. Consequently, $g$ is a homeomorphism and we let $h=g^{-1}$.

It remains to show that $f \circ h$ is continuous in the a.e. topology. We must show that for each open set $W,(f \circ h)^{-1}(W)$ is almost open. Recall that it is apparent (Proposition 2.2 in [3]) that if a set $U$ is almost open and has density 1 at a point $u$, then $U \cup\{u\}$ is almost open. Thus it will suffice to show that for each $u \in(f \circ h)^{-1}(W)$ there is an almost open set $G \subseteq(f \circ h)^{-1}(W)$ having density one at $u$. We claim that for each $u \in(f \circ h)^{-1}(W)$ there is an open (in the usual topology) set $G \subseteq(f \circ h)^{-1}(W)$ having density one at $u$, and the remainder of the proof consists of verifying that claim.

So, fix an open set $W$ and a $u \in(f \circ h)^{-1}(W)$. Let $y=h(u)$. If $y \in C(f)$, then our claim is clearly true. Suppose that $y \notin C(f)$. Then note that $y$ cannot belong to infinitely many of the $I_{n}$ 's since $\operatorname{osc} f \mid I_{n}<1 /(n+1)$. There is a subsequence $\left\{x_{n_{k}}\right\}$ of $\left\{x_{n}\right\}$ for which $R_{y} \backslash\{y\}=\left\{x_{n_{k}}\right\}$. Since $y$ only belongs to finitely many $I_{n_{k}}$, we let $K_{y}$ be the first index such that $y \notin \overline{I_{n_{k}}}$ for $k \geq K_{y}$, and set

$$
C_{y}=\bigcup_{k \geq K_{y}} I_{n_{k}} \cup\{y\} .
$$

Since $\lim _{k \rightarrow \infty} f\left(x_{n_{k}}\right)=f(y)$, we have that $\lim _{\substack{z \rightarrow y \\ z \in C_{y}}} f(z)=f(y)$; and thus to establish our claim it will suffice to show that $g\left(C_{y}\right)$ is the union of $\{u\}$ and an open set having density 1 at $u$. Since $g$ is a homeomorphism and $C_{y}$ is the union of $\{y\}$ and an open set, it is clear that $g\left(C_{y}\right)$ is the union of $\{u\}$ and an open set, $V$. It remains to show that $V$ has density 1 at $u$.

Consider the partition $Q_{n_{K y}}$. Since $Q_{n_{K y}} \subseteq C(f)$ and $y \notin C(f)$, there is a unique partition interval $\left[q_{n_{K_{y}}}, \hat{q}_{n_{K_{y}}}\right]$ for which $q_{n_{K_{y}}}<y<\hat{q}_{n_{K_{y}}}$. Consider the first $x_{m}$ such that $x_{m} \in\left(q_{n_{K_{y}}}, \hat{q}_{n_{K_{y}}}\right)$. Such an $x_{m}$ exists, $x_{m} \in R_{y}$ and $m>n_{K_{y}}$. Thus $I_{m} \subset C_{y}$. Then $x_{m} \in I_{m}=\left(a_{m}, b_{m}\right) \subset\left(q_{n_{K_{y}}}, \hat{q}_{n_{K_{y}}}\right)$ and $y \notin I_{m}$. In particular, $I_{m}$ lies entirely to one side of $y$. For definiteness and without loss of generality suppose that $I_{m}$ lies to the left of $y$. Recall how $g$ has been defined for the five points

$$
q_{n_{K_{y}}}<a_{m}<x_{m}<b_{m}<\hat{q}_{n_{K_{y}}} \text {. }
$$

First, $g$ was defined at $q_{n_{K_{y}}}$ and $\hat{q}_{n_{K_{y}}}$, but for no other points in $\left(q_{n_{K_{y}}}, \hat{q}_{n_{K_{y}}}\right)$ prior to being defined at $a_{m}, x_{m}$, and $b_{m}$. Letting $u_{n_{K_{y}}}=g\left(q_{n_{K_{y}}}\right)$ and $\hat{u}_{n_{K_{y}}}=$ $g\left(\hat{q}_{n_{K_{y}}}\right)$ we know that

$$
u_{n_{K_{y}}}<g\left(a_{m}\right)<g\left(x_{m}\right)<g\left(b_{m}\right)<\hat{u}_{n_{K_{y}}} .
$$


In particular, $g\left(x_{m}\right)$ is the midpoint of $\left(u_{n_{K_{y}}}, \hat{u}_{n_{K_{y}}}\right)$ and

$$
\frac{g\left(b_{m}\right)-g\left(a_{m}\right)}{\hat{u}_{n_{K_{y}}}-u_{n_{K_{y}}}} \geq 1-\frac{1}{2^{m+1}} \text {. }
$$

The point $u$ is in $\left(g\left(b_{m}\right), \hat{u}_{n_{K_{y}}}\right)$ and $g\left(I_{m}\right) \subseteq V$. So, let us get a first estimate of the density of $V$ at $u$. First consider those $z \in\left[u_{n_{K_{y}}}, g\left(a_{m}\right)\right]$ and the relative measure of $V$ in $[z, u]$. We have

$$
\frac{\lambda(V \cap[z, u])}{\lambda([z, u])}>\frac{\lambda\left(g\left(I_{m}\right)\right)}{\hat{u}_{n_{K_{y}}}-u_{n_{K_{y}}}} \geq 1-\frac{1}{2^{m+1}} .
$$

Next, since $\left(g\left(a_{m}\right), g\left(b_{m}\right)\right) \subseteq V$, it is clear that for all $z \in\left(g\left(a_{m}\right), g\left(b_{m}\right)\right)$ we have

$$
\frac{\lambda(V \cap[z, u])}{\lambda([z, u])} \geq \frac{\lambda\left(V \cap\left[g\left(b_{m}\right), u\right]\right)}{\lambda\left(\left[g\left(b_{m}\right), u\right]\right)} .
$$

Thus, we turn our attention to obtaining a bound on this latter relative measure.

Note that the partition interval containing $y$ based on the partition $Q_{m}$ is $\left[b_{m}, \hat{q}_{n_{K_{y}}}\right]$. This interval will not be refined until the first $x_{n}$ lands in $\left(b_{m}, \hat{q}_{n_{K_{y}}}\right)$. Then $n>m$. We are interested in the left side of $y$, but there is nothing to prevent this $x_{n}$ from lying to the right of $y$. Furthermore, since $x_{m}<b_{m}$ we could have $x_{j}$ with $x_{m}<x_{j}<b_{m}, \quad n_{K_{y}}<m<j<n$. That is, we could have entries to $R_{y}^{-}$which lie in $\left(x_{m}, b_{m}\right)$. However, should this occur, we must have $I_{j} \subset I_{m}$ by condition (b). Thus, $g\left(I_{j}\right) \subseteq g\left(I_{m}\right) \subseteq V$; that is, $I_{j}$ adds no more measure to $V$ than we were aware of when we considered $g\left(I_{m}\right)$. Thus, we ignore these $I_{j}$ 's. Now, suppose that our $x_{n}$ does, indeed, lie on the right side of $y$. Then $y$ is left of $I_{n}$ and the partition interval from the partition $Q_{n}$ containing $y$ is $\left[b_{m}, a_{n}\right]$ with $y<a_{n}$. Now we wait for the first entry, say $x_{n^{*}}$, into $\left(b_{m}, a_{n}\right)$. Again, it might be on the right side of $y$. If so, we move on to the partition interval of $Q_{n^{*}}$ which contains $y$, namely $\left[b_{m}, a_{n^{*}}\right]$, with $y<a_{n^{*}}$. We continue until we encounter the first $x_{s}$ which lands to the left of $y$. Note that the partition interval from the partition $Q_{s-1}$ which contains $y$ will be $\left[b_{m}, \hat{q}_{s-1}\right]$, and we know that $y<\hat{q}_{s-1}$. Thus we have encountered a situation basically the same as the one we started with where we had $y$ in the partitioning interval $\left(q_{n_{K_{y}}}, \hat{q}_{n_{K_{y}}}\right)$ and $I_{m}$ to the left of $y$. Presently, we have $y$ contained in the partitioning interval $\left(b_{m}, \hat{q}_{s-1}\right)$ based on the partition $Q_{s-1}$ with $I_{s}=\left(a_{s}, b_{s}\right)$ lying in this interval and to the left of $y$. Note that we have $g\left(I_{s}\right) \subseteq V, g\left(I_{s}\right) \subseteq\left[g\left(b_{m}\right), u\right]$, $\left[g\left(b_{m}\right), u\right] \subseteq\left[g\left(b_{m}\right), g\left(\hat{q}_{s-1}\right)\right], g\left(x_{s}\right)$ the midpoint of $\left[g\left(b_{m}\right), g\left(\hat{q}_{s-1}\right)\right]$, and

$$
\frac{\lambda\left(g\left(I_{s}\right)\right)}{g\left(\hat{q}_{s-1}\right)-g\left(b_{m}\right)} \geq 1-\frac{1}{2^{s+1}} \text {. }
$$

Again, since $g\left(I_{s}\right) \subset V$, this implies that

$$
\frac{\lambda\left(V \cap\left[g\left(b_{m}\right), u\right]\right)}{\lambda\left(\left[g\left(b_{m}\right), u\right]\right)}>1-\frac{1}{2^{s+1}}>1-\frac{1}{2^{m+1}} .
$$

Thus, to this point, we have that for all $z \in\left(g\left(a_{m}\right), g\left(b_{m}\right)\right]$,

$$
\frac{\lambda(V \cap[z, u])}{\lambda([z, u])} \geq 1-\frac{1}{2^{m+1}},
$$


and for all $z \in\left(g\left(b_{m}\right), g\left(a_{s}\right)\right)$

$$
\frac{\lambda(V \cap[z, u])}{\lambda([z, u])} \geq 1-\frac{1}{2^{s+1}} .
$$

For $z \in\left[g\left(a_{s}\right), g\left(b_{s}\right)\right]$ we again see that our relative measure is bounded below by the relative measure obtained when $z=g\left(b_{s}\right)$. When we consider $\left[g\left(b_{s}\right), u\right]$ we go through exactly the same process as before. We ignore those left returns to $y$ which are less than $g\left(b_{s}\right)$. We use the right returns to $y$ which come before the first left return closer than $g\left(b_{s}\right)$ to move up $Q$ 's partition interval closer to $y$ on the right. Then we again estimate with the first left return, $x_{t}$, to $y$ closer to $y$ than $g\left(b_{s}\right)$. Our relative measure estimate will then be $1-1 / 2^{t+1}>1-1 / 2^{s+1}$. Consequently, we will be able to show that $V$ has left density 1 at $u$, and a similar argument will show that $V$ has right density 1 at $u$ as well, completing the proof that $(3) \Rightarrow(4)$.

Finally, we show that $(4) \Rightarrow(1)$ : Since $f \circ h$ is continuous in the a.e. topology, it readily follows that $f$ is Baire 1 , Darboux. It remains to show that $f \mid C(f)$ is dense in $f$. To this end let $x \in[0,1]$ and let $V$ be a neighborhood of $f(x)$. Also since $f \circ h$ is continuous in the a.e. topology, $(f \circ h)^{-1}(V)$ has density one at $h^{-1}(x)$, and hence there are points of $C(f \circ h)$ arbitrarily close to $h^{-1}(x)$ in $(f \circ h)^{-1}(V)$. Since $h$ is a homeomorphism, $C(f)$ has points arbitrarily close to $x$ in $f^{-1}(V)$, showing that $f \in \mathscr{G}$ and thereby completing the proof of this theorem.

The authors wish to express their gratitude to the referee, whose thoughtful suggestions have improved the exposition of this work.

\section{REFERENCES}

1. U. B. Darji, M. J. Evans, and R. J. O'Malley, First return path systems: differentiability, continuity, and orderings, Acta. Hungar. (to appear).

2. I. Maximoff, Sur la transformation continue de quelques fonctions en derivees exactes, Bull. Soc. Phys. Math. Kazan (3) 12 (1940), 57-81.

3. R. J. O'Malley, Approximately continuous functions which are continuous almost everywhere, Acta Math. Acad. Sci. Hungar. 33 (1979), 395-402.

4. __ First return path derivatives, Proc. Amer. Math. Soc. 116 (1992), 73-77.

5. D. Preiss, Maximoff's Theorem, Real Anal. Exchange 5 (1979), 92-104.

(U. B. Darji and M. J. Evans) Department of Mathematics, North Carolina State UniVersity, Raleigh, North Carolina 27695-8205

Current address, U. B. Darji: Department of Mathematics, University of Louisville, Louisville, Kentucky 40292

E-mail address, U. B. Darji: ubdarj01@ulkyvx. louisville.edu

Current address, M. J. Evans: Department of Mathematics, Washington and Lee University, Lexington, Virginia 24450

E-mail address, M. J. Evans: mjevans@wlu.edu

(R. J. O'Malley) Department of Mathematical Sciences, University of WisconsinMilwaukee, Milwaukee, Wisconsin 53201

E-mail address, R. J. O’Malley: omalley@csd4.csd.uwm.edu 